\title{
Title: Factors that coordinate mesoderm specification from neuromesodermal progenitors with segmentation during vertebrate axial extension
}

\author{
Benjamin L. Martin \\ Department of Biochemistry and Cell Biology \\ Stony Brook University \\ Stony Brook, NY 11794-5215
}

benjamin.martin@stonybrook.edu

\begin{abstract}
The formation of the vertebrate body depends on the precise timing and coordination of molecular and morphological events. During vertebrate embryogenesis, the paraxial mesoderm is segmented into structures called somites in a progressive fashion from the anterior to the posterior at the same time as the entire body axis elongates in the posterior direction. Evidence from several vertebrate species indicates that new paraxial mesoderm is continuously induced from neuromesodermal progenitors at the posterior-most end of the embryo. The newly forming mesoderm exists in a specialized environment called the mesodermal progenitor niche. This review will discuss how the progenitor niche coordinates the continuous addition of new mesoderm to the body axis with proper segmentation of this mesoderm upon exit from the niche. I


will focus on evidence that the t-box transcription factor Brachyury and its downstream transcriptional targets serve as the primary factors coordinating mesoderm specification with somitogenesis. I will end with a discussion of recent exciting work regarding the cell-cycle and migratory behavior of mesodermal cells as they exit the progenitor niche, which may serve to further integrate new mesoderm production with proper segmentation.

Keywords: Axial extension, somitogenesis, mesodermal progenitor niche, neuromesodermal progenitors, Brachyury, Wnt

\section{Introduction}

During vertebrate embryogenesis, the head forms first and the rest of the body forms progressively away from the head. This process leads to a wave of differentiation along the anterior/posterior axis, with anterior cells differentiating first and posterior cells added continuously to the elongating tissues [1]. The progressive differentiation of the body axis is best visualized by observing somitogenesis, the process by which the paraxial mesoderm segments and differentiates into pairs of somites at regular intervals from the anterior to the posterior [2]. The continuous formation of somites requires the sustained contribution of new cells to the paraxial presomitic mesoderm (PSM), which are provided by a population of posteriorly localized progenitors [1]. There is now strong evidence from several vertebrate species that paraxial mesoderm continues to be generated from bipotential neuromesodermal progenitors (NMPs) throughout the entire course of somite formation and axis extension (Fig. 1A, B) [3-9]. During commitment to 
the mesodermal lineage from NMPs, cells exist in a niche (also referred to as the mesodermal maturation zone) that has defined molecular and cellular properties (Fig. 1A, B) $[4,10,11]$. This review will focus on how the properties of the mesodermal progenitor niche coordinate both the sustained specification and maintenance of mesodermal progenitors with the proper segmental patterning of the paraxial mesoderm.

\section{A brief overview of the clock and wavefront model of somitogenesis}

In order to understand how the molecular and cellular properties of the mesodermal progenitor niche affect somitogenesis, it is necessary to provide a basic overview of the molecular mechanism governing this process. For a more in-depth appreciation of the molecular control of somitogenesis, there are some excellent recent reviews [2, 12-14]. In all vertebrates, somites form at regular intervals (the exact timing of which varies between species) in an anterior to posterior progression from unsegmented PSM. As cells leave the anterior PSM and become incorporated into somites, new cells join the posterior PSM as the embryo undergoes posterior axial elongation [14-16]. The clock and wavefront model is used to describe the molecular mechanism of somitogenesis [14-17] (Fig. 1C). The clock represents molecular on-off oscillations of gene expression in somite progenitor cells. Each cell is capable of maintaining oscillating gene expression independently, and neighboring cells become synchronized in their oscillations through the Delta/Notch signaling pathway [18-23]. Many of the cycling genes are themselves members of the Delta/Notch signaling pathway, including the hairy enhancer of split related transcription factors (abbreviated her in zebrafish and Hes in mouse and chick) and the Notch receptor ligand Delta [14-16]. In mouse and chick but not in zebrafish, 
components of the canonical Wnt (hereafter referred to as Wnt) and FGF pathways also cycle in the PSM $[24,25]$. In the scope of this review I will focus specifically on components of the Delta/Notch signaling pathway with respect to cycling genes. The conserved importance of Delta/Notch signaling during somitogenesis is reflected in the fact that disruptions in this signaling pathway cause somite defects in all vertebrate embryos in which it has been examined, including mice, chick, Xenopus, and zebrafish $[16,26]$. Significantly, disruptions in this pathway also cause human vertebral defects leading to congenital scoliosis $[16,27,28]$. The oscillations in gene expression move from the posterior to the anterior PSM at a defined rate (depending on the species), giving the impression of a "wave" of gene expression [15] (Fig. 1). In the anterior-most PSM, the stripe of expression stops oscillating and provides the temporal and spatial information for somite border formation [15]. The wavefront (also called the determination front) is the anterior limit of signaling gradients across the somite progenitor field. The best characterized of these signals are the Wnt and FGF pathways, which are expressed in the posterior-most region of the embryo. As the embryo extends posteriorly, the signaling source and wavefront also move posteriorly. When cells are no longer within a threshold Wnt and FGF activity level, they become competent to respond to the signal generated by the segmentation clock and activate mesoderm posterior genes (Mesp2 in mouse and mespb in zebrafish). The Mesp proteins and their downstream targets orchestrate the physical boundary formation and a new somite forms $[29,30]$. An opposing gradient of retinoic acid signaling functions to inhibit Wnt and FGF signaling and helps position the wavefront [12]. As I will discuss further, many of the same signals 
and genes involved in the clock and wavefront mechanism of somitogenesis also have essential roles in generating new mesoderm from NMPs and during axial extension.

\section{Brachyury as a key integrator of mesoderm specification and somitogenesis}

Brachyury (abbreviated Bra, and also called T), which is Latin for "short tail," was the name given to a mutant line of mice, which exhibited truncated tails in the heterozygous state [31]. The gene responsible for this phenotype was later cloned and identified as the founding member of the t-box transcription factor family, for which there are 17 members in mouse and humans and 26 in zebrafish [32, 33]. Bra is expressed, along with the neural marker Sox2, in NMPs at the posterior-most end of the embryo in zebrafish, chick, mouse, and humans (as well as in in vitro derived NMPs), and is maintained for a short period in mesodermal progenitors after Sox2 is repressed [4, 34-38]. In both zebrafish and mouse (zebrafish and other teleost fish have two bra genes called $n t l a$ and $n t l b$, whereas mammals have a single gene), complete loss of Bra function results in embryonic lethality where embryos are severely truncated and only form the $1^{\text {st }}$ 8-12 somites (Fig. 2A, B) [39-42]. In all other vertebrates examined, loss of Bra function results in axial truncations, including naturally occurring Bra mutations, which are a common cause of taillessness in some species of dogs and cats when in the heterozygous state [43-46]. In addition to its striking and well-conserved role in mesoderm specification and axial extension across vertebrate species, Bra also appears to be an integral component of the clock and wavefront mechanism of somitogenesis. The 8-12 somites that form in zebrafish and mouse Bra loss of function embryos are poorly formed with irregular borders, suggesting a role in proper somitogenesis that is distinct from its 
role in mesoderm specification (Fig. 2B) [39, 40]. Consistent with a role in somitogenesis, a human congenital scoliosis study found that a missense mutation in $B R A$ is statistically enriched in patients [47]. In the following sections, I will discuss the direct transcriptional targets of Brachyury that facilitate its function in both mesoderm specification from NMPs and somitogenesis.

\section{Bra establishes a niche environment of high Wnt and low retinoic acid signaling}

Stem or progenitor cell niches in embryonic or adult organisms typically consist of a bipartite cellular arrangement that includes distinct cell types including both support cells and the stem or progenitor cells [48-50]. The support cells can contribute to the physical and molecular properties of the niche such as direct cell adhesion to the progenitors or the secretion of signaling factors that can promote or inhibit differentiation. The mesodermal progenitor population is unique in that the progenitor cells also serve as the support population by creating a signaling environment that can facilitate mesodermal development of cells lacking key mesodermal genes [10].

Bra serves as the key regulator of the molecular signaling environment of the mesodermal progenitor niche. Bra directly activates Wnt ligand transcription in zebrafish, mouse, and human, and Wnt signaling in turn directly regulates Bra expression, thereby creating a positive autoregulatory loop [40, 51-53]. Bra also directly activates the transcription of the cytochrome p450 26a1 enzyme (cyp26al), which degrades retinoic acid [10]. Together, these Bra target genes create an environment in the mesodermal progenitors of high Wnt signaling and low retinoic acid signaling. Importantly, in zebrafish, individual cells in the mesodermal progenitor niche that lack the ability to 
express bra (ntla and ntlb), Wnt ligands wnt8a and wnt $3 a$, and cyp26al, can develop normally when surrounded by wild-type mesodermal progenitors, indicating that the progenitors also serve as the support cells. This is in stark contrast to the whole embryo loss of function phenotypes of these genes, where the entire signaling environment of the mesodermal progenitors breaks down and the progenitors are not sustained, leading to truncated embryos with fewer somites than wild-type animals [54-60]. In addition to their role in sustaining mesodermal progenitors, the Wnt and retinoic acid signaling pathways also have critical functions during the segmentation process through regulation of the clock and wavefront mechanism, which will be discussed in more detail below (Fig. 2D).

\section{Wnt signaling specifies new mesoderm and affects the clock and wavefront during somitogenesis}

Wnt signaling has a dynamic role in both the specification of new PSM and the segmentation process, affecting cell fate and migration in the niche, and both aspects of the clock and wavefront activities during segmentation (Fig. 2D). The posterior localization of Wnt signaling during axial extension is a conserved feature of metazoans $[61,62]$. In vertebrates, posteriorly expressed wnt $3 a$ and $w n t 8 a$ ligands combine to induce new mesoderm, and the absence of these genes causes severe posterior truncations in the number of somites. The relative contributions of wnt3a and wnt $8 a$ to the formation of paraxial mesoderm varies between species, with wnt8a playing a greater role in earlier branching vertebrates such as fish and frogs, while wnt $3 a$ is more important for this process in mammals $[40,55,56,59,63-68]$. In all vertebrates, the loss of somites after the loss of Wnt signaling is accompanied by an increase in sox 2 positive neural 
progenitors, indicating that Wnt signaling plays a conserved role in the early induction of mesoderm during gastrulation $[4,36,37,51,57,69-73]$. In addition to its critical early role, wnt3a and/or wnt $8 a$ continue to be expressed after gastrulation in the posterior-most embryonic structure called the tailbud, with highest levels in the mesodermal progenitor population. During this sustained posterior expression, Wnt signaling continuously functions to induce new mesoderm from bipotential NMPs in the tailbud, which is recapitulated in NMP cell culture $[4,9,36,38,70,73]$. Together, these studies indicate that Wnt signaling within the mesodermal progenitor niche has an essential function of inducing new mesoderm to join the progenitor pool from NMPs [9].

Wnt signaling promotes mesodermal fate in NMPs through regulation of the t-box transcription factor $T b x 6$. Mammals have a single $T b x 6$ gene, whereas zebrafish have three $T b x 6$ related genes; $t b x 16$ (also called spadetail), tbx6 (previously called $t b x 24$ and fused somites), and $t b x 6 l$ (previously called $t b x 6$ ) [32]. In zebrafish, tbx16 and $t b x 6 l$ are expressed in overlapping domains in the mesodermal progenitors, and $t b x 6$ is expressed slightly anterior in the PSM [74-76]. Wnt signaling promotes tbx16 expression, which in turn directly represses sox 2 and establishes the mesodermal fate decision from NMPs [77]. In mouse, Wnt signaling is necessary and sufficient for expression of the single Tbx6 gene [78], and loss of Tbx6 function results in an absence of posterior somites and an expansion of neural tissue in regions where somites should normally form $[79,80]$. The expansion of neural tissue in $T b x 6$ mutants is dependent on the failure to repress Sox2 in NMPs that exit into the paraxial mesoderm domain [81].

In addition to its pivotal role in mesoderm specification from NMPs, Wnt signaling plays a key role in the normal migration of cells from NMPs to mesodermal 
progenitors and finally to PSM. Time-lapse imaging of the zebrafish tailbud showed that NMPs in the dorsal medial zone of the tailbud undergo rapid, coordinated, and directional migration towards the posterior wall of the tailbud $[82,83]$. When Wnt signaling is reduced, these cells lose their coherence, causing disruptions in the normal migratory process, which can lead to shortening or bending of the body axis $[82,83]$. Further, the Wnt target genes Tbx6 and the bHLH transcription factor Msgnl in mouse, and $t b x 16$ and msgnl in zebrafish, play important roles in the exit of mesodermal progenitors into the PSM. The tbx16 mutant zebrafish was originally called spadetail, due to the fact that cells that normally exit the mesodermal progenitor zone into PSM remain stuck in the tailbud, creating a spade-like appearance $[74,84,85]$. In spadetail mutants, anterior somites fail to form, but tailbud exit recovers later in development and tail somites form relatively normally $[74,84,85]$. The exit of cells to form posterior somites in tbx16 mutants is dependent on msgnl function, and in the absence of both tbxl6 and msgnl a larger spade forms and all somites are absent [86-88]. In mouse, loss of either Tbx6 or Msgn1 function causes a spadetail-like phenotype, with mesodermal progenitors failing to exit the niche properly and accumulating in the tailbud [80, 89]. Interestingly, zebrafish msgnl mutants form extra somites that are smaller than normal, which is predicted if the somite clock oscillation rate is the same but fewer cells enter the PSM during each oscillation cycle [86]. This is consistent with Wnt signaling controlling the rate of progenitor exit into the PSM [77]. Based on these studies, it is clear that all new mesoderm that joins the PSM throughout axis extension must be induced by high Wnt signaling, providing a unique opportunity for this pathway to pattern the PSM into highly ordered segments. 
While Wnt signaling induces new mesoderm and promotes migration and mesodermal progenitor exit into the PSM during axial extension, it also has direct and indirect roles in the molecular clock and wavefront (Fig. 2D). Wnt signaling activity, which is highest in the posterior tailbud domain as it induces new mesoderm, exists in a posterior to anterior gradient [2]. High Wnt signaling levels maintain Notch signaling pathway oscillations in the PSM. Below a threshold Wnt activity, Notch pathway oscillations cease, mesp is expressed, and somite formation occurs [90]. In zebrafish, a recent report indicates that Wnt activity ceases posterior to segment formation, indicating that a threshold response of Wnt activity is not directly responsible for initiating segment formation in this species [91]. Instead the Wnt target genes msgn1, tbx6l, and tbx16 may transduce the signal for somite formation [91]. Msgn1 and Tbx6 are also direct Wnt target genes in mouse, and in addition to their critical roles in Wnt mediated mesoderm specification and exit from the progenitor niche, also have essential roles in somitogenesis. Tbx6, which is expressed in the mesodermal progenitors and PSM in mouse, is required, in conjunction with Notch signaling, for the activation of Mesp2 expression and somite border formation [92]. Although Tbx6 loss of function mice lack most paraxial mesoderm and therefore also lack most somites [80], a hypomorphic Tbx6 allele called rib vertebrae ( $r b$ ) forms paraxial mesoderm but fails to segment $[93,94]$. Similarly, zebrafish lacking function of the $T b x 6$-related gene $t b x 6$, which is expressed in the PSM, form paraxial mesoderm but fail to activate mespb and form segments, despite normal cycling of herl [76]. On the other hand, mouse Msgnl activates the Notch pathway and is required for expression of cycling genes in the PSM [95]. Together, the Wnt target genes tbx6 and msgn1, which induce new mesoderm and promote its exit from 
the niche into the PSM, simultaneously help establish both the wavefront and clock during somitogenesis.

\section{Cyp26a1 protects the Brachyury/Wnt autoregulatory loop and helps establish the wavefront}

Retinoic acid is generated in the posterior-most somites, where the retinoic acid synthesizing enzyme aldhla2 is expressed $[96,97]$. Despite being in relatively close proximity to the posterior somite retinoic acid source, the mesodermal progenitor niche is an environment of low retinoic acid, achieved through expression of the retinoic acid metabolizing enzyme cyp26al [98-100]. In zebrafish, cyp26al transcription is directly activated by Bra (ntla) [10]. The juxtaposition of the RA source and sink creates a linear gradient of RA across the PSM, which can be observed by direct RA visualization [101]. In the absence of cyp26al function in both mouse and zebrafish, RA signaling expands into the posterior-most region of the embryo and causes truncation of the axis with fewer than normal somites forming $[54,60]$. This is similar to the axis truncation effect of elevated RA signaling caused by exogenous RA addition [10, 102]. It is essential that mesodermal progenitors exist in a low RA environment because RA signaling is a potent repressor of bra (ntla) expression, which when repressed causes a breakdown in the $\mathrm{Bra} / \mathrm{Wnt}$ autoregulatory loop, thus causing a loss of mesoderm specification and creating axis truncations $[10,34]$. Therefore, Bra protects its own expression by directly regulating cyp26al expression to prevent RA mediated repression, and simultaneously helps establish a linear RA gradient across the PSM (Fig. 2D). 
RA signaling plays an essential role in the positioning of the wavefront during somitogenesis. In addition to its role in repressing Wnt ligand expression, RA signaling represses FGF signaling, including direct repression of $F g f 8$ transcription, one of the two ligands ( $F g f 4$ being the other) involved in establishing the FGF wavefront in mouse [103, 104]. Since the FGF activity gradient in the PSM is established through decay of ligand mRNA, repression of FGF ligand expression directly impinges upon the location of the wavefront [105]. A similar decay mechanism may occur with regard to the Wnt gradient, as both Wnt and FGF ligands are transcribed in the mesodermal progenitors and not in the PSM [2]. Accordingly, loss of RA signaling (leading to an anterior shift of the wavefront) causes smaller somite formation [106]. The situation in zebrafish appears different, as RA positively regulate $f g f 8$ and $f g f 17$ expression [107], although like amniotes the absence of RA still results in smaller somites [108, 109].

\section{Other direct Bra target genes linking mesoderm specification with somitogenesis}

Bra is clearly required for the continued generation of new mesoderm across vertebrates, but it also appears to regulate dynamics of the somitogenesis clock, as the 812 somites that form after complete loss of Bra function in mouse and zebrafish have poorly formed borders (Fig. 2) [39, 40]. While Wnt signaling directly regulates Bra expression, and loss of Wnt function also causes a loss of posterior somites, the anterior somites that form in Wnt loss of function embryos have well formed somite boundaries $[4,51,56,57,59]$. This is likely due to the fact that while Wnt3a/Wnt8a mediated Wnt signaling directly regulates $\mathrm{Bra}$ expression, it is required for maintenance of $\mathrm{Bra}$ expression rather than the initial induction during gastrula stages, which instead is 
regulated by Nodal, BMP, and FGF signaling [4, 110-116]. This suggests that Bra regulates somitogenesis at least in part independently of its regulation of Wnt signaling. What Bra target genes might mediate this activity? As previously mentioned, Notch signaling is a critical conserved component of the somitogenesis molecular clock across vertebrate species, and disruptions of the Notch ligand Delta function causes defects in somitogenesis. In zebrafish, Bra (Ntla) directly regulates both deltaC and deltaD expression [53, 117, 118], and deltaC (originally called beamter) and deltaD (originally called after eight) mutants have severe somitogenesis defects [119-121]. Similarly, in mouse, Bra regulates Dll1 expression, and t-box binding sites in Dll1 regulatory sequence are essential for its expression $[122,123]$. Dll1 homozygous mutants have severe disruptions in the formation of all somites [124]. Expression of Delta ligands is also dependent on Wnt signaling and the Wnt target gene Tbx6, so loss of expression is likely compounded by the loss of Bra and Wnt signaling in Bra mutants, due to breakdown of the Bra/Wnt autoregulatory loop [78, 122].

The Her/HES transcriptional repressors, which serve as core oscillating components of the clock and function within the Notch pathway, are also directly regulated by Bra. In zebrafish, Bra (Ntla) binding was detected in $-9 \mathrm{~kb}$ to $+3 \mathrm{~kb}$ region of both herl and her7 [53], two genes known to be critical for proper somitogenesis [125127]. herl has at least one functional t-box binding site required for transcriptional activation, and her7 has one of the largest clusters of consensus t-box binding sites of any gene in the zebrafish genome (although untested functionally) $[118,128]$. In mouse, Hes7 is an oscillating gene of the segmentation clock and loss of function results in failure of proper somitogenesis $[129,130]$. Hes7 is a direct BRA target in human and mouse [131]. 
These results indicate that both the Notch ligand Delta and the hairy enhancer of split family of genes involved in the somitogenesis clock are conserved Bra target genes across vertebrates. Therefore, Bra directly influences somitogenesis independent of its role in mesoderm specification (Fig. 2).

\section{Cellular behavior in the progenitor niche - is it noisy in there?}

In the clock and wavefront model, individual cells function as independent molecular oscillators. In order for proper somitogenesis to occur, these independent oscillators must be locally entrained to oscillate synchronously, which is accomplished through Delta/Notch signaling [19]. Newly specified mesoderm of the tailbud exhibits behaviors traditionally expected to produce noise in the clock and wavefront model, most notably rapid cell migration and cell division. But recent mathematical modeling and experimental manipulations indicate that these noisy behaviors, to a certain extent, may actually promote molecular clock entrainment rather than inhibit it.

Mathematical models can accurately predict the molecular oscillations that lead to the regular, sequential formation of somites during vertebrate body formation $[18,132$, 133]. However, a flaw in many models is the discrepancy between predicted recovery and actual recovery in embryos of molecular oscillations in neighboring cells after transient disruption of cellular synchrony through Delta/Notch signaling inhibition [134]. In mathematical models it would take tens to hundreds of oscillations to recover synchrony, whereas experimental data in zebrafish shows that the embryo can recover synchrony in neighboring cells and proper somite formation within ten oscillations [21, 134]. A revised mathematical model can correct this discrepancy by introducing random 
cell movement as cells first begin their oscillations [134]. In the model, the molecular oscillations in neighboring cells, which are synchronized by direct cell-to-cell contact through Delta/Notch signaling, can synchronize rapidly in a group of cells if they increase the number of cells they contact through random cell movement (Fig. 3B) [134]. Random movement and mixing in mesodermal progenitor cells occurs in chick embryos [135]. The random migration in these cells is controlled by FGF signaling, and promotes the axial extension of the developing embryo. In zebrafish, cells in the mesodermal progenitor niche are highly motile, mix readily with each other, and display unsynchronized clock oscillations compared to their neighbor cells. As cells join the posterior PSM, motility and mixing decrease substantially, and oscillatory gene expression appears synchronized between neighbors [20]. Therefore, the rapid and seemingly random mixing of newly specified mesodermal progenitor cells may play a dual role in coordinating axial extension with molecular oscillator entrainment needed for proper somite formation (Fig. 3B). Further experiments are required to determine whether mathematical predications of cell mixing based clock entrainment between neighbor cells occurs in vivo.

Another putative source of noise during the entrainment of molecular oscillators of the somite clock is cell division. In zebrafish, NMPs of the tailbud are mostly held in the G2 stage of the cell cycle, with relatively few mitotic events [136, 137]. As NMPs are induced to form mesoderm and join the mesodermal progenitor pool, they undergo a transit-amplifying step where they turn on the expression of $c d c 25 a$, a factor that promotes entry into mitosis [136]. The concerted expression of $c d c 25 a$ in cells entering the mesodermal progenitor population causes a synchronized entry into mitosis and a 
population doubling [136]. The direct effect of this mitotic event on the cycling of the molecular oscillator of the somite clock was visualized using a transgenic reporter system in zebrafish that allows the direct observation of cycling gene expression, using the herl promoter to drive a Her1-Venus fusion protein [138]. Interestingly, the two daughter cells produced after a cell division event have highly entrained cycling reporter expression with each other, even in the absence of Notch signaling where oscillations between neighbor cells tend to be asynchronous. This suggests that equal distribution of molecular components of the oscillator in daughter cells entrains them for future oscillations. Furthermore, cytokinesis occurred predominantly during the off phase of the reporter oscillation, at a time when neighboring cells were also in the off-phase, which minimized the effect of noise between dividing and non-dividing cells [138]. The combined data from these two studies that mesodermal progenitors undergo a synchronous division when they first join the progenitor pool, and that daughter cells have highly entrained clock oscillations, suggests that the initial cell division event shortly after cells acquire a mesodermal identity may actually function to entrain a population of cells, rather than produce noise (Fig. 3C). In support of this model, zebrafish embryos that have a mutation in emil, which prevents cells from progressing from G2 to M phase after the gastrula stage, have a greater degree of asynchronous herl expression than wild-type embryos at the 15 somite stage, which results in irregular and disorganized somite borders [139]. It will be interesting to see whether other vertebrate species exhibit synchronized mitoses in newly specified mesoderm.

\section{Conclusions:}


The growing body of literature regarding the properties of progenitor cells in the posterior end of vertebrate embryos has shed light on the deeply integrated processes of continuous mesoderm specification and segmentation, with many of the same genes and signaling pathways mediating both events. The Bra/Wnt autoregulatory loop is a key pathway orchestrating these processes, directly affecting mesoderm specification, cell migration into and out of the mesodermal progenitor niche, and various aspects of both the molecular clock and wavefront during segmentation. It is becoming increasingly clear that many of the factors discussed in this review, in particular Wnt signaling, also integrate other aspects of body patterning. Wnt signaling is involved in regulating Hox gene expression via caudal homeobox genes $(c d x)$ genes, which impart axial identity (such as whether a segment forms a cervical, thoracic, or lumbar vertebra) to segments $[61,140]$. Hox gene expression also controls exit from the mesodermal progenitor niche, and posterior Hox gene expression has recently been shown to negatively regulate Wnt signaling, which subsequently causes posterior axial elongation to cease [141-143]. Thus, the continued investigation of the molecules and cellular properties of the NMPs and mesodermal progenitor niche will shed light on how apparently disparate morphological and patterning events are actually intricately intertwined to globally regulate body plan development during embryogenesis.

\section{Acknowledgements}

I would like to thank Dave Matus and Richard Row for comments on the manuscript, and members of the lab for helpful discussions. Support comes from Stony Brook University, 
The American Heart Association (13SDG14360032), and the National Science Foundation (IOS1452928).

\section{Figure 1 - Mesoderm specification from NMPs is concomitant with somitogenesis.}

(A, B) A lateral (A) and ventral (B) view of an embryo illustrating the relative position of cell compartments. Wnt signaling induces new mesoderm from NMPs and promotes exit into the paraxial mesoderm. (C) Four time points are shown during the formation of one somite. Individual cells exhibit oscillating gene expression (indicated by blue color for the "on" phase of the cycle), which are coordinated with neighboring cells. This coordination creates the appearance of stripes of expression, and as they cycle, gives the impression of a traveling wave of gene expression. Cells in the anterior stripe of expression cease their oscillations and remain in the "on" state, and where expression correlates with the signaling wavefront, a new somite border is formed ( $\mathrm{S} 1=$ most recently formed somite). While this process is occurring, the embryo is extending in the posterior direction. The schematics are from a ventral view with anterior to the top.

\section{Figure 2 - Brachyury and its target genes integrate mesoderm specification with} somitogenesis. (A) A wild-type mouse or zebrafish forms 60 or 33 somites respectively. (B) After complete loss of Brachyury in both mouse and zebrafish, the axis is truncated and embryos have only 8-12 poorly formed somites. (C) In vertebrates, Brachyury is expressed in the NMPs and mesodermal progenitors, as well as the notochord (blue). (D) Direct and secondary targets of Brachyury that control mesoderm specification and segment formation (see text for more details of specific target gene function). 
Figure 3-Cell behaviors of newly specified mesoderm hypothesized to coordinate somite clock oscillatory coupling. (A) A schematic showing the transition site from mesodermal progenitor (yellow) to PSM (red). (B) Random cell mixing in chick embryos is controlled by FGF signaling and promotes axial extension. Mixing increases cell contacts, which is predicted in mathematical models to entrain oscillation states of a group of cells through Delta/Notch signaling. (C) In zebrafish embryos, activation of $c d c 25 a$ expression in mesodermal progenitors causes synchronized entry into mitosis, and daughter cells maintain highly entrained oscillatory states with each other. Shades of blue represent oscillatory states of her/Hes expression.

[1] Wilson V, Olivera-Martinez I, Storey KG. Stem cells, signals and vertebrate body axis extension. Development 2009;136:1591-604.

[2] Hubaud A, Pourquie O. Signalling dynamics in vertebrate segmentation. Nature reviews Molecular cell biology 2014;15:709-21.

[3] Davis RL, Kirschner MW. The fate of cells in the tailbud of Xenopus laevis. Development 2000;127:255-67.

[4] Martin BL, Kimelman D. Canonical Wnt Signaling Dynamically Controls Multiple Stem Cell Fate Decisions during Vertebrate Body Formation. Developmental Cell 2012;22:223-32.

[5] Tzouanacou E, Wegener A, Wymeersch FJ, Wilson V, Nicolas J-F. Redefining the Progression of Lineage Segregations during Mammalian Embryogenesis by Clonal Analysis. Developmental Cell 2009;17:365-76.

[6] Freese NH, Lam BA, Staton M, Scott A, Chapman SC. A Novel Gain-Of-Function Mutation of the Proneural IRX1 and IRX2 Genes Disrupts Axis Elongation in the Araucana Rumpless Chicken. Plos One 2014;9.

[7] Gentsch GE, Owens NDL, Martin SR, Piccinelli P, Faial T, Trotter MWB, et al. In Vivo T-Box Transcription Factor Profiling Reveals Joint Regulation of Embryonic Neuromesodermal Bipotency. Cell Reports 2013;4:1185-96.

[8] Cambray N, Wilson V. Axial progenitors with extensive potency are localised to the mouse chordoneural hinge. Development 2002;129:4855-66.

[9] Henrique D, Abranches E, Verrier L, Storey KG. Neuromesodermal progenitors and the making of the spinal cord. Development 2015;142:2864-+.

[10] Martin BL, Kimelman D. Brachyury establishes the embryonic mesodermal progenitor niche. Genes \& Development 2010;24:2778-83. 
[11] Griffin KJP, Kimelman D. One-Eyed Pinhead and Spadetail are essential for heart and somite formation. Nature Cell Biology 2002;4:821-5.

[12] Aulehla A, Pourquie O. Signaling gradients during paraxial mesoderm development. Cold Spring Harb Perspect Biol 2010;2:a000869.

[13] Maroto M, Bone RA, Dale JK. Somitogenesis. Development 2012;139:2453-6.

[14] Oates AC, Morelli LG, Ares S. Patterning embryos with oscillations: structure, function and dynamics of the vertebrate segmentation clock. Development 2012;139:62539.

[15] Mara A, Holley SA. Oscillators and the emergence of tissue organization during zebrafish somitogenesis. Trends Cell Biol 2007;17:593-9.

[16] Pourquie O. Vertebrate Segmentation: From Cyclic Gene Networks to Scoliosis. Cell 2011;145:650-63.

[17] Cooke J, Zeeman EC. A clock and wavefront model for control of the number of repeated structures during animal morphogenesis. J Theor Biol 1976;58:455-76.

[18] Horikawa K, Ishimatsu K, Yoshimoto E, Kondo S, Takeda H. Noise-resistant and synchronized oscillation of the segmentation clock. Nature 2006;441:719-23.

[19] Jiang YJ, Aerne BL, Smithers L, Haddon C, Ish-Horowicz D, Lewis J. Notch signalling and the synchronization of the somite segmentation clock. Nature 2000;408:475-9.

[20] Mara A, Schroeder J, Chalouni C, Holley SA. Priming, initiation and synchronization of the segmentation clock by deltaD and deltaC. Nat Cell Biol 2007;9:523-30.

[21] Riedel-Kruse IH, Muller C, Oates AC. Synchrony dynamics during initiation, failure, and rescue of the segmentation clock. Science 2007;317:1911-5.

[22] Ozbudak EM, Lewis J. Notch signalling synchronizes the zebrafish segmentation clock but is not needed to create somite boundaries. PLoS Genet 2008;4:e15.

[23] Okubo Y, Sugawara T, Abe-Koduka N, Kanno J, Kimura A, Saga Y. Lfng regulates the synchronized oscillation of the mouse segmentation clock via trans-repression of Notch signalling. Nat Commun 2012;3:1141.

[24] Dequeant ML, Glynn E, Gaudenz K, Wahl M, Chen J, Mushegian A, et al. A complex oscillating network of signaling genes underlies the mouse segmentation clock.

Science 2006;314:1595-8.

[25] Krol AJ, Roellig D, Dequeant ML, Tassy O, Glynn E, Hattem G, et al. Evolutionary plasticity of segmentation clock networks. Development 2011;138:2783-92.

[26] Rida PC, Le Minh N, Jiang YJ. A Notch feeling of somite segmentation and beyond. Dev Biol 2004;265:2-22.

[27] Sparrow DB, Chapman G, Dunwoodie SL. The mouse notches up another success: understanding the causes of human vertebral malformation. Mamm Genome 2011;22:362-76.

[28] Eckalbar WL, Fisher RE, Rawls A, Kusumi K. Scoliosis and segmentation defects of the vertebrae. Wiley interdisciplinary reviews Developmental biology 2012;1:401-23. [29] Saga Y. The mechanism of somite formation in mice. Curr Opin Genet Dev 2012;22:331-8.

[30] Saga Y, Hata N, Koseki H, Taketo MM. Mesp2: a novel mouse gene expressed in the presegmented mesoderm and essential for segmentation initiation. Genes Dev 1997;11:1827-39. 
[31] Dobrovolskaia-Zavadskaia N. Regarding the spontaneous mortification of the tail of a new-born mouse and the existence of a hereditary characteristic (factor). Comptes Rendus Des Seances De La Societe De Biologie Et De Ses Filiales 1927;97:114-6. [32] Ahn D, You KH, Kim CH. Evolution of the Tbx6/16 Subfamily Genes in Vertebrates: Insights from Zebrafish. Molecular Biology and Evolution 2012;29:3959-83. [33] Herrmann BG, Labeit S, Poustka A, King TR, Lehrach H. Cloning of the T-gene required in mesoderm formation in the mouse. Nature 1990;343:617-22.

[34] Olivera-Martinez I, Harada H, Halley PA, Storey KG. Loss of FGF-Dependent Mesoderm Identity and Rise of Endogenous Retinoid Signalling Determine Cessation of Body Axis Elongation. Plos Biology 2012;10.

[35] Delfino-Machin M, Lunn JS, Breitkreuz DN, Akai J, Storey KG. Specification and maintenance of the spinal cord stem zone. Development 2005;132:4273-83.

[36] Gouti M, Tsakiridis A, Wymeersch FJ, Huang Y, Kleinjung J, Wilson V, et al. In vitro generation of neuromesodermal progenitors reveals distinct roles for wnt signalling in the specification of spinal cord and paraxial mesoderm identity. PLoS Biol 2014;12:e1001937.

[37] Tsakiridis A, Huang Y, Blin G, Skylaki S, Wymeersch F, Osorno R, et al. Distinct Wnt-driven primitive streak-like populations reflect in vivo lineage precursors.

Development 2015;142:809.

[38] Garriock RJ, Chalamalasetty RB, Kennedy MW, Canizales LC, Lewandoski M, Yamaguchi TP. Lineage tracing of neuromesodermal progenitors reveals novel Wntdependent roles in trunk progenitor cell maintenance and differentiation. Development 2015;142:1628-38.

[39] Chesley P. Development of the short-tailed mutant in the house mouse. Journal of Experimental Zoology 1935;70:429-59.

[40] Martin BL, Kimelman D. Regulation of canonical Wnt signaling by Brachyury is essential for posterior mesoderm formation. Developmental Cell 2008;15:121-33.

[41] Halpern ME, Ho RK, Walker C, Kimmel CB. Induction of muscle pioneers and floor plate is distinguished by the zebrafish no tail mutation. Cell 1993;75:99-111.

[42] Schultemerker S, Vaneeden FJM, Halpern ME, Kimmel CB, Nussleinvolhard C. no tail (ntl) is the zebrafish homolog of the mouse-T (Brachyury) gene. Development 1994;120:1009-15.

[43] Buckingham KJ, McMillin MJ, Brassil MM, Shively KM, Magnaye KM, Cortes A, et al. Multiple mutant $\mathrm{T}$ alleles cause haploinsufficiency of Brachyury and short tails in Manx cats. Mammalian genome : official journal of the International Mammalian Genome Society 2013;24:400-8.

[44] Conlon FL, Sedgwick SG, Weston KM, Smith JC. Inhibition of Xbra transcription activation causes defects in mesodermal patterning and reveals autoregulation of Xbra in dorsal mesoderm. Development 1996;122:2427-35.

[45] Haworth K, Putt W, Cattanach B, Breen M, Binns M, Lingaas F, et al. Canine homolog of the T-box transcription factor T; failure of the protein to bind to its DNA target leads to a short-tail phenotype. Mammalian genome : official journal of the International Mammalian Genome Society 2001;12:212-8.

[46] Hytonen MK, Grall A, Hedan B, Dreano S, Seguin SJ, Delattre D, et al. Ancestral Tbox mutation is present in many, but not all, short-tailed dog breeds. The Journal of heredity 2009;100:236-40. 
[47] Ghebranious N, Blank RD, Raggio CL, Staubli J, McPherson E, Ivacic L, et al. A missense T (Brachyury) mutation contributes to vertebral malformations. J Bone Miner Res 2008;23:1576-83.

[48] Morrison SJ, Spradling AC. Stem cells and niches: Mechanisms that promote stem cell maintenance throughout life. Cell 2008;132:598-611.

[49] Scadden DT. Nice neighborhood: emerging concepts of the stem cell niche. Cell 2014; 157:41-50.

[50] Voog J, Jones DL. Stem Cells and the Niche: A Dynamic Duo. Cell Stem Cell 2010;6:103-15.

[51] Yamaguchi TP, Takada S, Yoshikawa Y, Wu NY, McMahon AP. T (Brachyury) is a direct target of Wnt3a during paraxial mesoderm specification. Genes \& Development 1999;13:3185-90.

[52] Evans AL, Faial T, Gilchrist MJ, Down T, Vallier L, Pedersen RA, et al. Genomic targets of Brachyury (T) in differentiating mouse embryonic stem cells. PLoS One 2012; 7:e33346.

[53] Morley RH, Lachani K, Keefe D, Gilchrist MJ, Flicek P, Smith JC, et al. A gene regulatory network directed by zebrafish No tail accounts for its roles in mesoderm formation. Proc Natl Acad Sci U S A 2009;106:3829-34.

[54] Abu-Abed S, Dolle P, Metzger D, Beckett B, Chambon P, Petkovich M. The retinoic acid-metabolizing enzyme, CYP26A1, is essential for normal hindbrain patterning, vertebral identity, and development of posterior structures. Genes \& Development 2001;15:226-40.

[55] Lekven AC, Thorpe CJ, Waxman JS, Moon RT. Zebrafish wnt8 encodes two wnt8 proteins on a bicistronic transcript and is required for mesoderm and neurectoderm patterning. Developmental Cell 2001;1:103-14.

[56] Shimizu T, Bae YK, Muraoka O, Hibi M. Interaction of Wnt and caudal-related genes in zebrafish posterior body formation. Developmental Biology 2005;279:125-41.

[57] Takada S, Stark KL, Shea MJ, Vassileva G, McMahon JA, McMahon AP. Wnt-3a regulates somite and tailbud formation in the mouse embryo. Genes \& Development 1994;8:174-89.

[58] Sakai Y, Meno C, Fujii H, Nishino J, Shiratori H, Saijoh Y, et al. The retinoic acidinactivating enzyme CYP26 is essential for establishing an uneven distribution of retinoic acid along the anterio-posterior axis within the mouse embryo. Genes \& Development 2001;15:213-25.

[59] Cunningham TJ, Kumar S, Yamaguchi TP, Duester G. Wnt8a and Wnt3a cooperate in the axial stem cell niche to promote mammalian body axis extension. Developmental Dynamics 2015;244:797-807.

[60] Emoto Y, Wada H, Okamoto H, Kudo A, Imai Y. Retinoic acid-metabolizing enzyme Cyp26a1 is essential tor determining territories of hindbrain and spinal cord in zebrafish. Developmental Biology 2005;278:415-27.

[61] Martin BL, Kimelman D. Wnt Signaling and the Evolution of Embryonic Posterior Development. Current Biology 2009;19:R215-R9.

[62] Petersen CP, Reddien PW. Wnt signaling and the polarity of the primary body axis. Cell 2009;139:1056-68.

[63] Baker KD, Ramel MC, Lekven AC. A Direct Role for Wnt8 in Ventrolateral Mesoderm Patterning. Developmental Dynamics 2010;239:2828-36. 
[64] Christian JL, McMahon JA, McMahon AP, Moon RT. Xwnt-8, a Xenopus Wnt1/int-1-related gene responsive to mesoderm-inducing growth-factors, may play a role in ventral mesodermal patterning during embryogenesis. Development 1991;111:1045-55. [65] Hoppler S, Brown JD, Moon RT. Expression of a dominant-negative Wnt blocks induction of MyoD in Xenopus embryos. Genes \& Development 1996;10:2805-17. [66] Hoppler S, Moon RT. BMP-2/-4 and Wnt-8 cooperatively pattern the Xenopus mesoderm. Mechanisms of Development 1998;71:119-29.

[67] Lu F-I, Thisse C, Thisse B. Identification and mechanism of regulation of the zebrafish dorsal determinant. Proceedings of the National Academy of Sciences of the United States of America 2011;108:15876-80.

[68] Wylie AD, Fleming J, Whitener AE, Lekven AC. Post-transcriptional regulation of wnt8a is essential to zebrafish axis development. Developmental Biology 2014;386:5363.

[69] Dunty WC, Kennedy MWL, Chalamalasetty RB, Campbell K, Yamaguchi TP. Transcriptional Profiling of Wnt3a Mutants Identifies Sp Transcription Factors as Essential Effectors of the Wnt/beta-catenin Pathway in Neuromesodermal Stem Cells. Plos One 2014;9.

[70] Jurberg AD, Aires R, Novoa A, Rowland JE, Mallo M. Compartment-dependent activities of Wnt3a/beta-catenin signaling during vertebrate axial extension. Dev Biol 2014;394:253-63.

[71] Nowotschin S, Ferrer-Vaquer A, Concepcion D, Papaioannou VE, Hadjantonakis A$\mathrm{K}$. Interaction of Wnt3a, Msgn1 and Tbx6 in neural versus paraxial mesoderm lineage commitment and paraxial mesoderm differentiation in the mouse embryo. Developmental Biology 2012;367:1-14.

[72] Tsakiridis A, Wilson V. Assessing the bipotency of in vitro-derived neuromesodermal progenitors. F1000Research 2015;4:100.

[73] Tsakiridis A, Huang Y, Blin G, Skylaki S, Wymeersch F, Osorno R, et al. Distinct Wnt-driven primitive streak-like populations reflect in vivo lineage precursors.

Development 2014;141:1209-21.

[74] Griffin KJP, Amacher SL, Kimmel CB, Kimelman D. Molecular identification of spadetail: regulation of zebrafish trunk and tail mesoderm formation by T-box genes.

Development 1998;125:3379-88.

[75] Hug B, Walter V, Grunwald DJ. tbx6, a Brachyury-related gene expressed by ventral mesendodermal precursors in the zebrafish embryo. Developmental Biology 1997;183:61-73.

[76] Nikaido M, Kawakami A, Sawada A, Furutani-Seiki M, Takeda H, Araki K. Tbx24, encoding a T-box protein, is mutated in the zebrafish somite-segmentation mutant fused somites. Nature Genetics 2002;31:195-9.

[77] Bouldin CM, Manning AJ, Peng YH, Farr GH, Hung KL, Dong A, et al. Wnt signaling and tbx16 form a bistable switch to commit bipotential progenitors to mesoderm. Development 2015;142:2499-+.

[78] Dunty WC, Jr., Biris KK, Chalamalasetty RB, Taketo MM, Lewandoski M, Yamaguchi TP. Wnt3a/beta-catenin signaling controls posterior body development by coordinating mesoderm formation and segmentation. Development 2008;135:85-94. 
[79] Chapman DL, Agulnik I, Hancock S, Silver LM, Papaioannou VE. Tbx6, a mouse T-box gene implicated in paraxial mesoderm formation at gastrulation. Developmental Biology 1996;180:534-42.

[80] Chapman DL, Papaioannou VE. Three neural tubes in mouse embryos with mutations in the T-box gene Tbx6. Nature 1998;391:695-7.

[81] Takemoto T, Uchikawa M, Yoshida M, Bell DM, Lovell-Badge R, Papaioannou VE, et al. Tbx6-dependent Sox2 regulation determines neural or mesodermal fate in axial stem cells. Nature 2011;470:394-8.

[82] Lawton AK, Nandi A, Stulberg MJ, Dray N, Sneddon MW, Pontius W, et al. Regulated tissue fluidity steers zebrafish body elongation. Development 2013;140:57382.

[83] McMillen P, Holley SA. The tissue mechanics of vertebrate body elongation and segmentation. Curr Opin Genet Dev 2015;32:106-11.

[84] Ho RK, Kane DA. Cell-autonomous action of zebrafish spt-1 mutation in specific mesodermal precursors. Nature 1990;348:728-30.

[85] Kimmel CB, Kane DA, Walker C, Warga RM, Rothman MB. A mutation that changes cell movement and cell fate in the zebrafish embryo. Nature 1989;337:358-62. [86] Fior R, Maxwell AA, Ma TP, Vezzaro A, Moens CB, Amacher SL, et al. The differentiation and movement of presomitic mesoderm progenitor cells are controlled by Mesogenin 1. Development 2012;139:4656-65.

[87] Yabe T, Takada S. Mesogenin causes embryonic mesoderm progenitors to differentiate during development of zebrafish tail somites. Developmental Biology 2012;370:213-22.

[88] Manning AJ, Kimelman D. Tbx16 and Msgn1 are required to establish directional cell migration of zebrafish mesodermal progenitors. Dev Biol 2015.

[89] Yoon JK, Wold B. The bHLH regulator pMesogenin 1 is required for maturation and segmentation of paraxial mesoderm. Genes Dev 2000;14:3204-14.

[90] Aulehla A, Wehrle C, Brand-Saberi B, Kemler R, Gossler A, Kanzler B, et al.

Wnt3A plays a major role in the segmentation clock controlling somitogenesis. Developmental Cell 2003;4:395-406.

[91] Bajard L, Morelli LG, Ares S, Pecreaux J, Julicher F, Oates AC. Wnt-regulated dynamics of positional information in zebrafish somitogenesis. Development 2014;141:1381-91.

[92] Yasuhiko Y, Haraguchi S, Kitajima S, Takahashi Y, Kanno J, Saga Y. Tbx6mediated Notch signaling controls somite-specific Mesp2 expression. Proc Natl Acad Sci U S A 2006;103:3651-6.

[93] Watabe-Rudolph M, Schlautmann N, Papaioannou VE, Gossler A. The mouse ribvertebrae mutation is a hypomorphic Tbx6 allele. Mech Dev 2002;119:251-6.

[94] White PH, Farkas DR, McFadden EE, Chapman DL. Defective somite patterning in mouse embryos with reduced levels of Tbx6. Development 2003;130:1681-90.

[95] Chalamalasetty RB, Dunty WC, Jr., Biris KK, Ajima R, Iacovino M, Beisaw A, et al. The Wnt3a/beta-catenin target gene Mesogenin1 controls the segmentation clock by activating a Notch signalling program. Nature Communications 2011;2.

[96] Dobbs-McAuliffe B, Zhao QS, Linney E. Feedback mechanisms regulate retinoic acid production and degradation in the zebrafish embryo. Mechanisms of Development 2004;121:339-50. 
[97] Niederreither K, McCaffery P, Drager UC, Chambon P, Dolle P. Restricted expression and retinoic acid-induced downregulation of the retinaldehyde dehydrogenase type 2 (RALDH-2) gene during mouse development. Mech Dev 1997;62:67-78.

[98] Fujii H, Sato T, Kaneko S, Gotoh O, Fujii-Kuriyama Y, Osawa K, et al. Metabolic inactivation of retinoic acid by a novel P450 differentially expressed in developing mouse embryos. The EMBO journal 1997;16:4163-73.

[99] de Roos K, Sonneveld E, Compaan B, ten Berge D, Durston AJ, van der Saag PT. Expression of retinoic acid 4-hydroxylase (CYP26) during mouse and Xenopus laevis embryogenesis. Mech Dev 1999;82:205-11.

[100] Swindell EC, Thaller C, Sockanathan S, Petkovich M, Jessell TM, Eichele G. Complementary domains of retinoic acid production and degradation in the early chick embryo. Dev Biol 1999;216:282-96.

[101] Shimozono S, Iimura T, Kitaguchi T, Higashijima S, Miyawaki A. Visualization of an endogenous retinoic acid gradient across embryonic development. Nature

2013;496:363-6.

[102] Sive HL, Draper BW, Harland RM, Weintraub H. Identification of a retinoic acidsensitive period during primary axis formation in Xenopus laevis. Genes \& Development 1990;4:932-42.

[103] Kumar S, Duester G. Retinoic acid controls body axis extension by directly repressing Fgf8 transcription. Development 2014;141:2972-7.

[104] Naiche LA, Holder N, Lewandoski M. FGF4 and FGF8 comprise the wavefront activity that controls somitogenesis. Proceedings of the National Academy of Sciences of the United States of America 2011;108:4018-23.

[105] Dubrulle J, Pourquie O. fgf8 mRNA decay establishes a gradient that couples axial elongation to patterning in the vertebrate embryo. Nature 2004;427:419-22.

[106] Diez del Corral R, Olivera-Martinez I, Goriely A, Gale E, Maden M, Storey K. Opposing FGF and retinoid pathways control ventral neural pattern, neuronal differentiation, and segmentation during body axis extension. Neuron 2003;40:65-79. [107] Hamade A, Deries M, Begemann G, Bally-Cuif L, Genet C, Sabatier F, et al. Retinoic acid activates myogenesis in vivo through Fgf8 signalling. Dev Biol 2006;289:127-40.

[108] Kawakami Y, Raya A, Raya RM, Rodriguez-Esteban C, Belmonte JCI. Retinoic acid signalling links left-right asymmetric patterning and bilaterally symmetric somitogenesis in the zebrafish embryo. Nature 2005;435:165-71.

[109] Begemann G, Schilling TF, Rauch GJ, Geisler R, Ingham PW. The zebrafish neckless mutation reveals a requirement for raldh2 in mesodermal signals that pattern the hindbrain. Development 2001;128:3081-94.

[110] Griffin K, Patient R, Holder N. Analysis of Fgf function in normal and no tail zebrafish embryos reveals separate mechanisms for formation of the trunk and the tail. Development 1995;121:2983-94.

[111] Harvey SA, Tumpel S, Dubrulle J, Schier AF, Smith JC. no tail integrates two modes of mesoderm induction. Development 2010;137:1127-35.

[112] Latinkic BV, Umbhauer M, Neal KA, Lerchner W, Smith JC, Cunliffe V. The Xenopus Brachyury promoter is activated by FGF and low concentrations of activin and suppressed by high concentrations of activin and by paired-type homeodomain proteins. Genes Dev 1997;11:3265-76. 
[113] Smith JC, Price BM, Green JB, Weigel D, Herrmann BG. Expression of a Xenopus homolog of Brachyury $(\mathrm{T})$ is an immediate-early response to mesoderm induction. Cell 1991;67:79-87.

[114] Feldman B, Gates MA, Egan ES, Dougan ST, Rennebeck G, Sirotkin HI, et al. Zebrafish organizer development and germ-layer formation require nodal-related signals. Nature 1998;395:181-5.

[115] Conlon FL, Lyons KM, Takaesu N, Barth KS, Kispert A, Herrmann B, et al. A primary requirement for nodal in the formation and maintenance of the primitive streak in the mouse. Development 1994;120:1919-28.

[116] Winnier G, Blessing M, Labosky PA, Hogan BL. Bone morphogenetic protein-4 is required for mesoderm formation and patterning in the mouse. Genes Dev 1995;9:210516.

[117] Jahangiri L, Nelson AC, Wardle FC. A cis-regulatory module upstream of deltaC regulated by Ntla and Tbx 16 drives expression in the tailbud, presomitic mesoderm and somites. Developmental Biology 2012;371:110-20.

[118] Garnett AT, Han TM, Gilchrist MJ, Smith JC, Eisen MB, Wardle FC, et al. Identification of direct T-box target genes in the developing zebrafish mesoderm. Development 2009;136:749-60.

[119] Holley SA, Geisler R, Nusslein-Volhard C. Control of her1 expression during zebrafish somitogenesis by a Delta-dependent oscillator and an independent wave-front activity. Genes \& Development 2000;14:1678-90.

[120] Julich D, Hwee Lim C, Round J, Nicolaije C, Schroeder J, Davies A, et al. beamter/deltaC and the role of Notch ligands in the zebrafish somite segmentation, hindbrain neurogenesis and hypochord differentiation. Dev Biol 2005;286:391-404. [121] vanEeden FJM, Granato M, Schach U, Brand M, FurutaniSeiki M, Haffter P, et al. Mutations affecting somite formation and patterning in the zebrafish, Danio rerio. Development 1996;123:153-64. [122] Hofmann M, Schuster-Gossler K, Watabe-Rudolph M, Aulehla A, Herrmann BG, Gossler A. WNT signaling, in synergy with T/TBX6, controls Notch signaling by regulating DII1 expression in the presomitic mesoderm of mouse embryos. Genes \& Development 2004;18:2712-7.

[123] Concepcion D, Papaioannou VE. Nature and extent of left/right axis defects in T(Wis) /T(Wis) mutant mouse embryos. Dev Dyn 2014;243:1046-53.

[124] Hrabe de Angelis M, McIntyre J, 2nd, Gossler A. Maintenance of somite borders in mice requires the Delta homologue DII1. Nature 1997;386:717-21.

[125] Henry CA, Urban MK, Dill KK, Merlie JP, Page MF, Kimmel CB, et al. Two linked hairy/Enhancer of split-related zebrafish genes, her1 and her7, function together to refine alternating somite boundaries. Development 2002;129:3693-704.

[126] Holley SA, Julich D, Rauch GJ, Geisler R, Nusslein-Volhard C. her1 and the notch pathway function within the oscillator mechanism that regulates zebrafish somitogenesis. Development 2002;129:1175-83.

[127] Oates AC, Ho RK. Hairy/E(spl)-related (Her) genes are central components of the segmentation oscillator and display redundancy with the Delta/Notch signaling pathway in the formation of anterior segmental boundaries in the zebrafish. Development 2002;129:2929-46. 
[128] Brend T, Holley SA. Expression of the oscillating gene her1 is directly regulated by Hairy/Enhancer of Split, T-box, and Suppressor of Hairless proteins in the zebrafish segmentation clock. Dev Dyn 2009;238:2745-59.

[129] Bessho Y, Hirata H, Masamizu Y, Kageyama R. Periodic repression by the bHLH factor Hes7 is an essential mechanism for the somite segmentation clock. Genes Dev 2003;17:1451-6.

[130] Bessho Y, Miyoshi G, Sakata R, Kageyama R. Hes7: a bHLH-type repressor gene regulated by Notch and expressed in the presomitic mesoderm. Genes to cells : devoted to molecular \& cellular mechanisms 2001;6:175-85.

[131] Faial T, Bernardo AS, Mendjan S, Diamanti E, Ortmann D, Gentsch GE, et al. Brachyury and SMAD signalling collaboratively orchestrate distinct mesoderm and endoderm gene regulatory networks in differentiating human embryonic stem cells. Development 2015;142:2121-+.

[132] Lewis J. Autoinhibition with transcriptional delay: a simple mechanism for the zebrafish somitogenesis oscillator. Curr Biol 2003;13:1398-408.

[133] Tiedemann HB, Schneltzer E, Zeiser S, Rubio-Aliaga I, Wurst W, Beckers J, et al. Cell-based simulation of dynamic expression patterns in the presomitic mesoderm. $\mathbf{J}$

Theor Biol 2007;248:120-9.

[134] Uriu K, Morishita Y, Iwasa Y. Random cell movement promotes synchronization of the segmentation clock. Proc Natl Acad Sci U S A 2010;107:4979-84.

[135] Benazeraf B, Francois P, Baker RE, Denans N, Little CD, Pourquie O. A random cell motility gradient downstream of FGF controls elongation of an amniote embryo.

Nature 2010;466:248-52.

[136] Bouldin CM, Snelson CD, Farr GH, Kimelman D. Restricted expression of cdc25a in the tailbud is essential for formation of the zebrafish posterior body. Genes \& Development 2014;28:384-95.

[137] Kanki JP, Ho RK. The development of the posterior body in zebrafish.

Development 1997;124:881-93.

[138] Delaune EA, Francois P, Shih NP, Amacher SL. Single-Cell-Resolution Imaging of the Impact of Notch Signaling and Mitosis on Segmentation Clock Dynamics.

Developmental Cell 2012;23:995-1005.

[139] Zhang L, Kendrick C, Julich D, Holley SA. Cell cycle progression is required for zebrafish somite morphogenesis but not segmentation clock function. Development 2008;135:2065-70.

[140] Kimelman D, Martin BL. Anterior-posterior patterning in early development: three strategies. Wiley Interdisciplinary Reviews-Developmental Biology 2012;1:253-66.

[141] Denans N, limura T, Pourquie O. Hox genes control vertebrate body elongation by collinear Wnt repression. Elife 2015;4.

[142] Iimura T, Pourquie O. Collinear activation of Hoxb genes during gastrulation is linked to mesoderm cell ingression. Nature 2006;442:568-71.

[143] Young T, Rowland JE, van de Ven C, Bialecka M, Novoa A, Carapuco M, et al. Cdx and Hox Genes Differentially Regulate Posterior Axial Growth in Mammalian Embryos. Developmental Cell 2009;17:516-26. 
progenitors

Mesodermal progenitors

Paraxial mesoderm

Spinal cord

A Anterior $\longrightarrow$ Posterior
Neuromesodermal progenitors

()

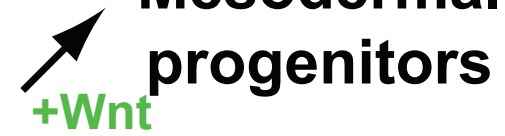
mesoderm

-Wnt

\section{Spinal cord}

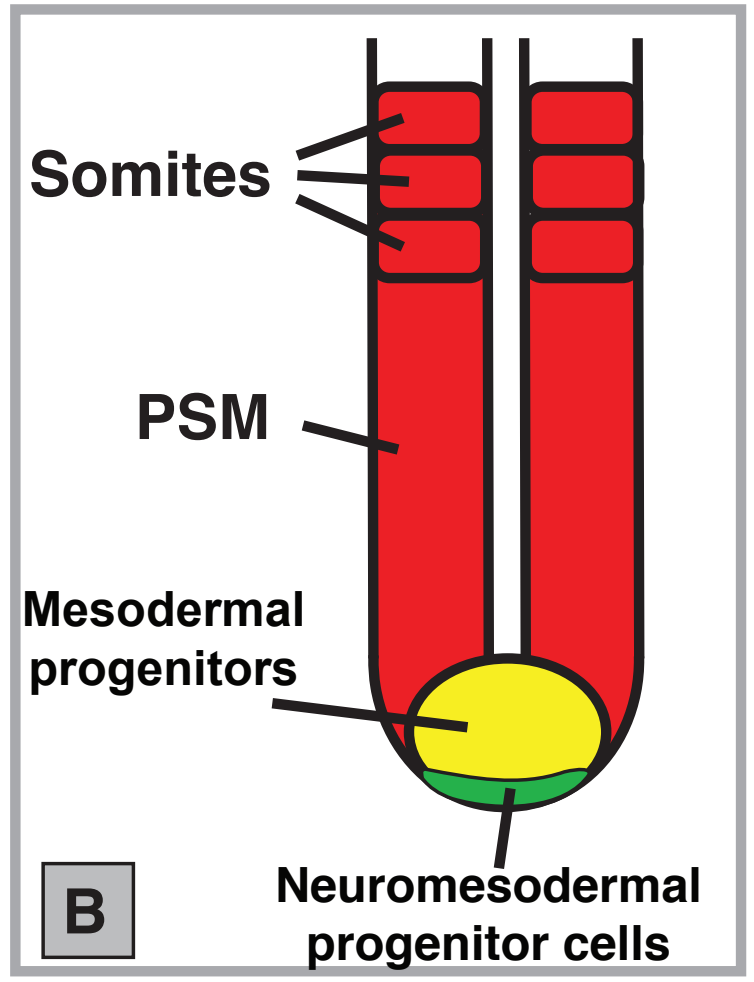

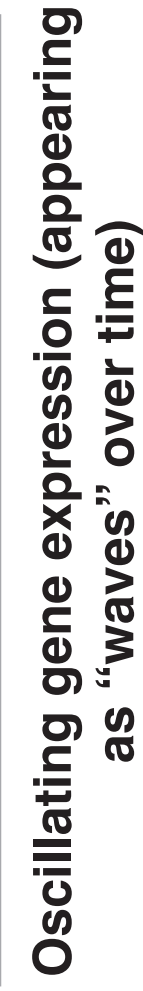

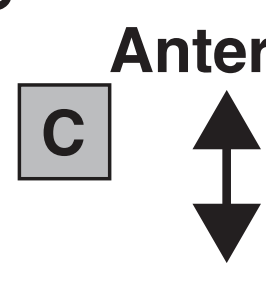

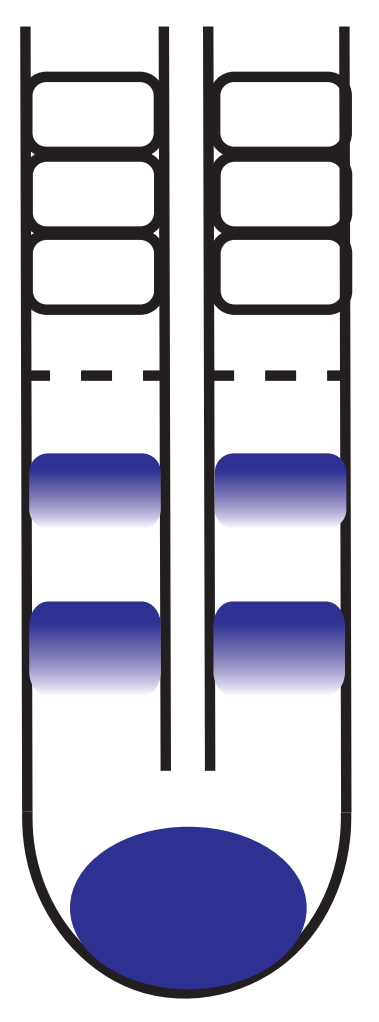
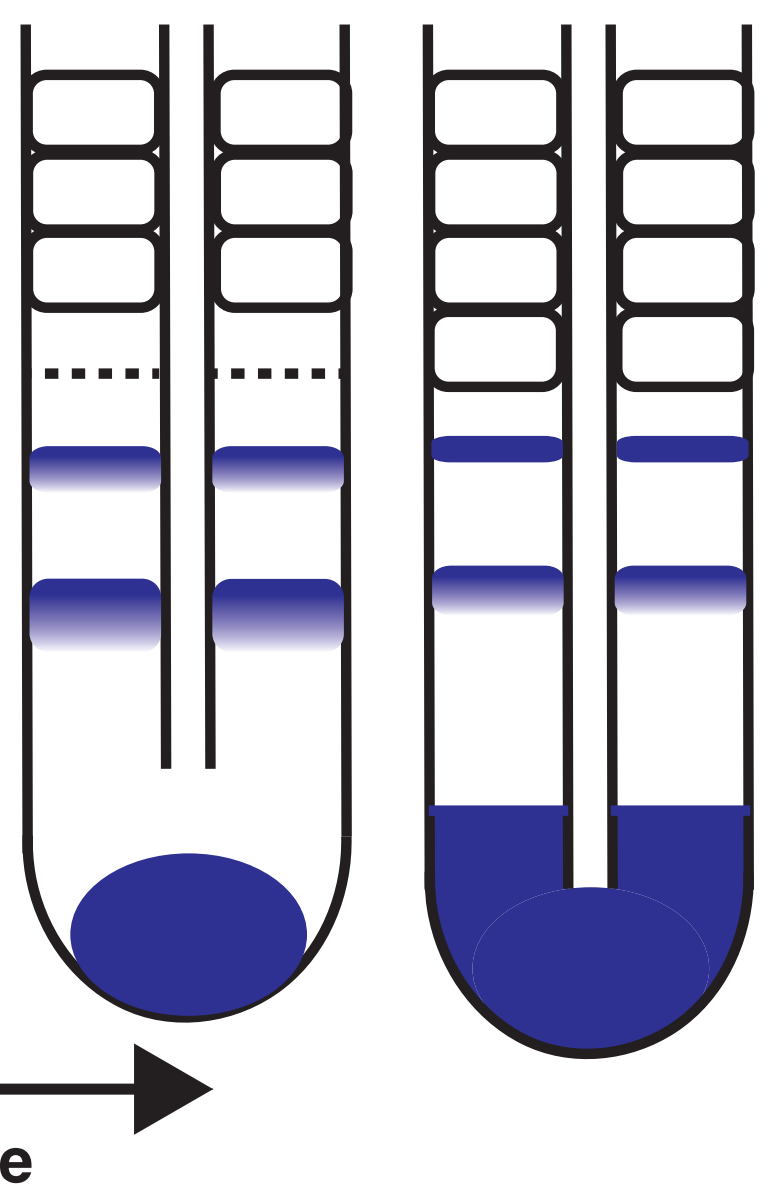

S2

S1

so

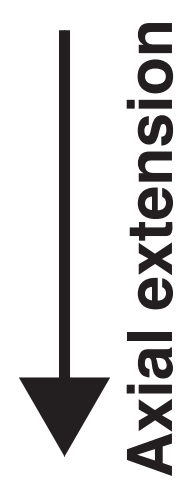

\section{Posterior}


Wild-type Bra LOF

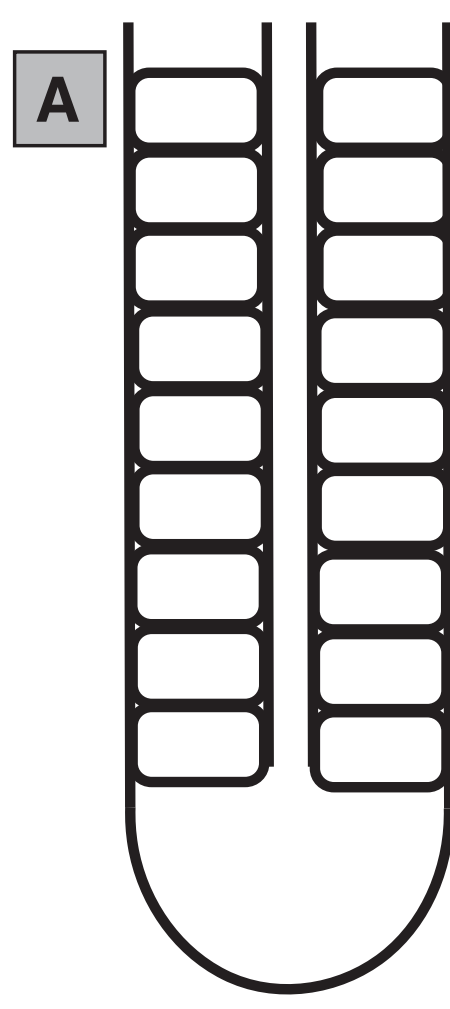

Bra expression

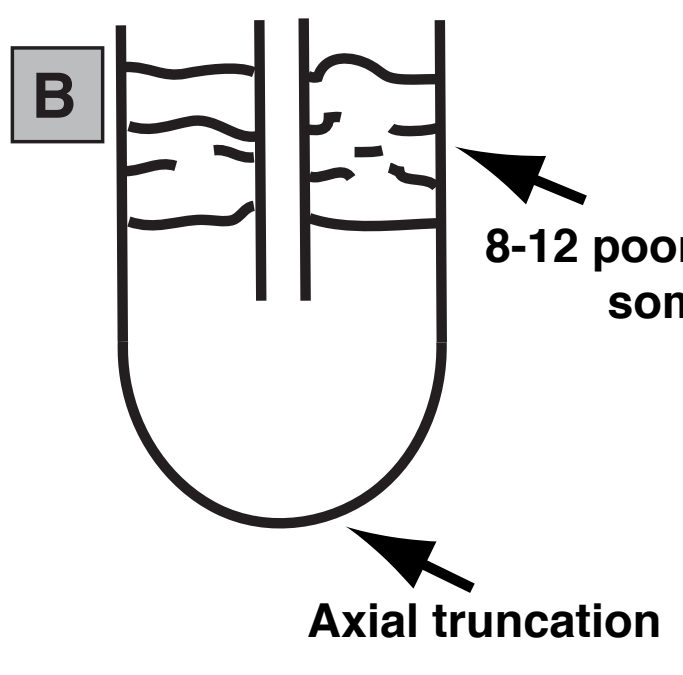

pattern

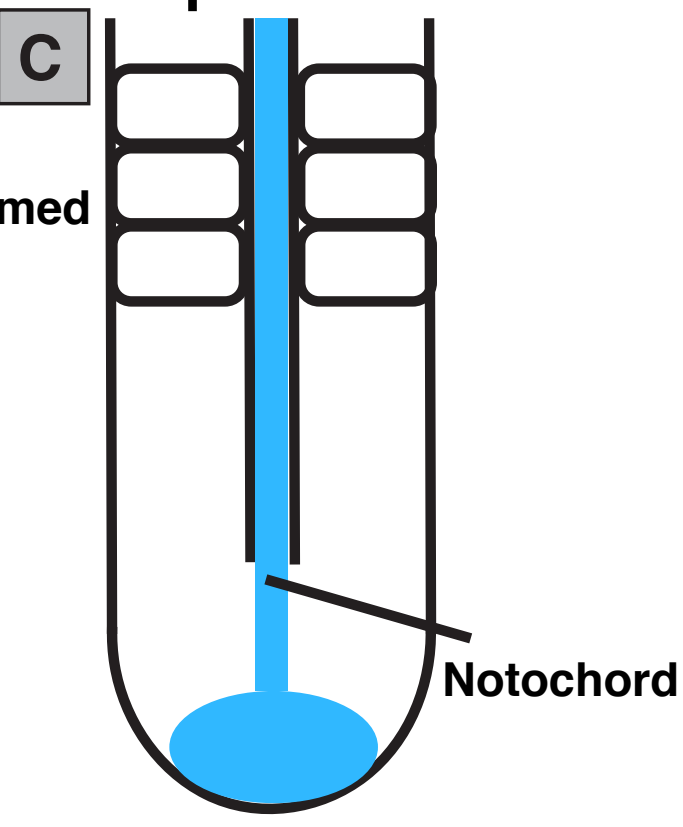

$1^{\circ}$ Bra target genes

\begin{abstract}
Mesoderm specification from NMPs
\end{abstract}

Canonical Wnt ligands

cyp26a1
Wavefront

Canonical Wnt ligands

cyp26a1
Molecular clock

Canonical Wnt ligands

Delta

her/Hes

$2^{\circ}$ Bra target genes via Wnt activation

$t b \times 6 / t b \times 16 / t b \times 6 \mid$

msgn1 tbx6/tbx $16 / t b \times 6 I$ msgn1

Delta

her/Hes 


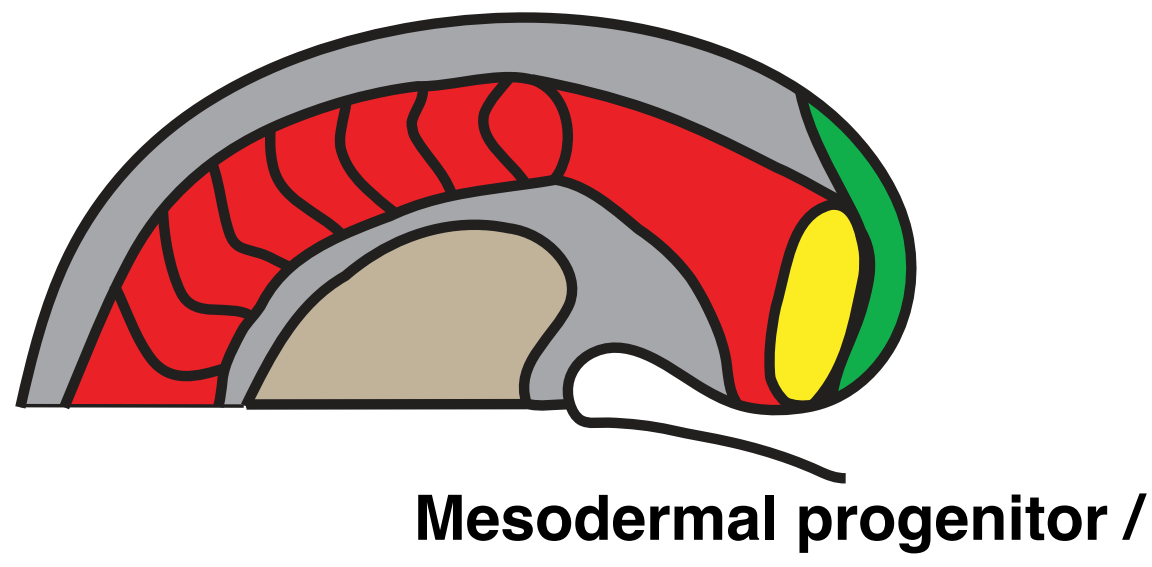

PSM posterior-most PSM
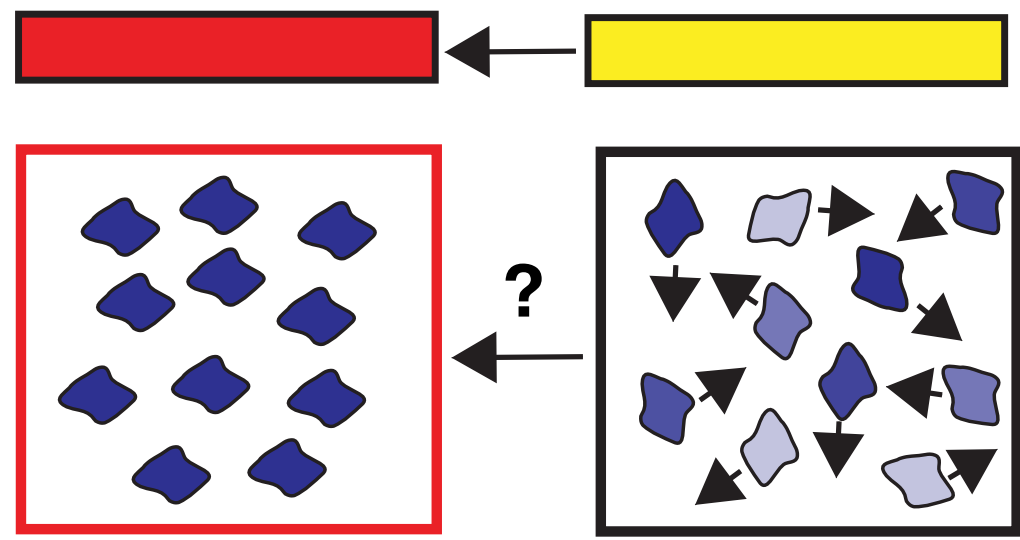

A
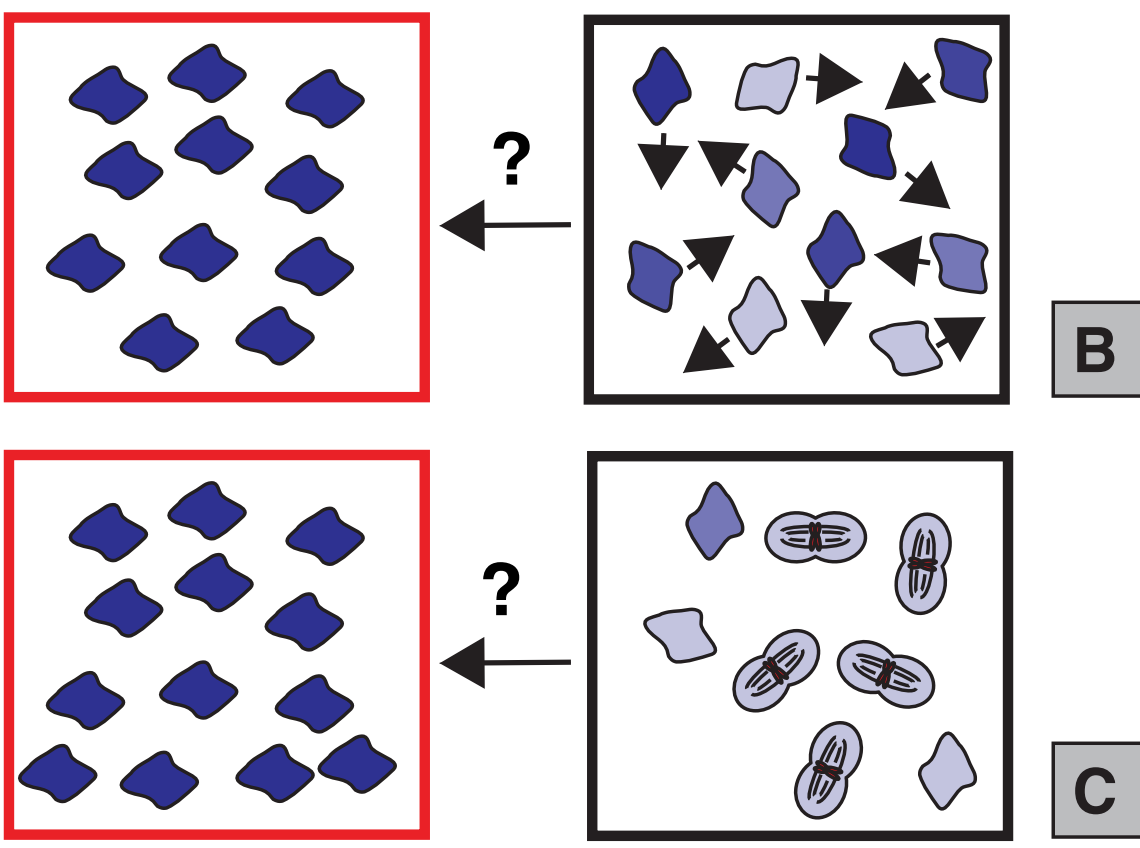

C

her/Hes oscillation state

Figure - 3 